\title{
MICROBIAL TRANSFORMATION OF CYCLOARTENOL AND 24-METHYLENE CYCLOARTANOL
}

\author{
K. C. Wang, * Liang-Hwa Young, Yu Wang, † and Shoei-Sheng Loe \\ School of Pharmacy, College of Medicine, and ${ }^{\dagger}$ NSC X-ray Center, Department of Chemistry, \\ National Taiwan University, Taipei 10018, Taiwan, R.O.C.
}

Summary: Cycloartenol (I) and 24-methylenecycloartanol (II) are the major triterpene alcohols present in the rice bran oil. Upon exposure of I, II and lanosterol to the growing culture of Mycobacterium sp. (NRRL B-3805), androsta-4,8(14)-diene-3, 17-dione (III) was isolated and characterized as the major metabolite in 34\%, 35\% and $30 \%$ yield, respectively.

It is well known that a wide range of microorganisms are able to utilize cholesterol and phytosterols as the sole carbon source for growth and propagation. 1,2 It is also well documented that in the steroid degrading microorganisms, the ring structure and side chain are metabolized by different enzyme systems simultaneously and independently. ${ }^{3}$ In recent years, methods for selective cleavage of the sterol side chains by microorganisms, such as (i) modification of sterol structure, ${ }^{4}$ (ii) addition of enzyme inhibitors, ${ }^{5-7}$ and (iii) employment of mutants, $8-9$ were developed to give $\mathrm{C}_{19}$ and/ or other useful steroid intermediates. The brilliant work of Sih and coworkers 10 led us gain an insight into the pathways and mechanisms involved in the microbial degradation of sterol side chains.

Cycloartenol (I) and 24-methylenecycloartanol (II) are the major triterpene alcohols present in the rice bran oil. 11 In both compounds, the C-19 carbon is incorporated into a cyclopropyl ring with C-9 and C-10 carbons and there are three methyl groups located at 4-,4- and 14a-positions of steroid nucleus. Upon exposure of compound I and II to the growing culture of Mycobacterium sp (NRRL B-3805), 8,12 compound III was isolated from the incubation mixture as the major metabolite in $34 \%$ and $35 \%$ yield, respectively.

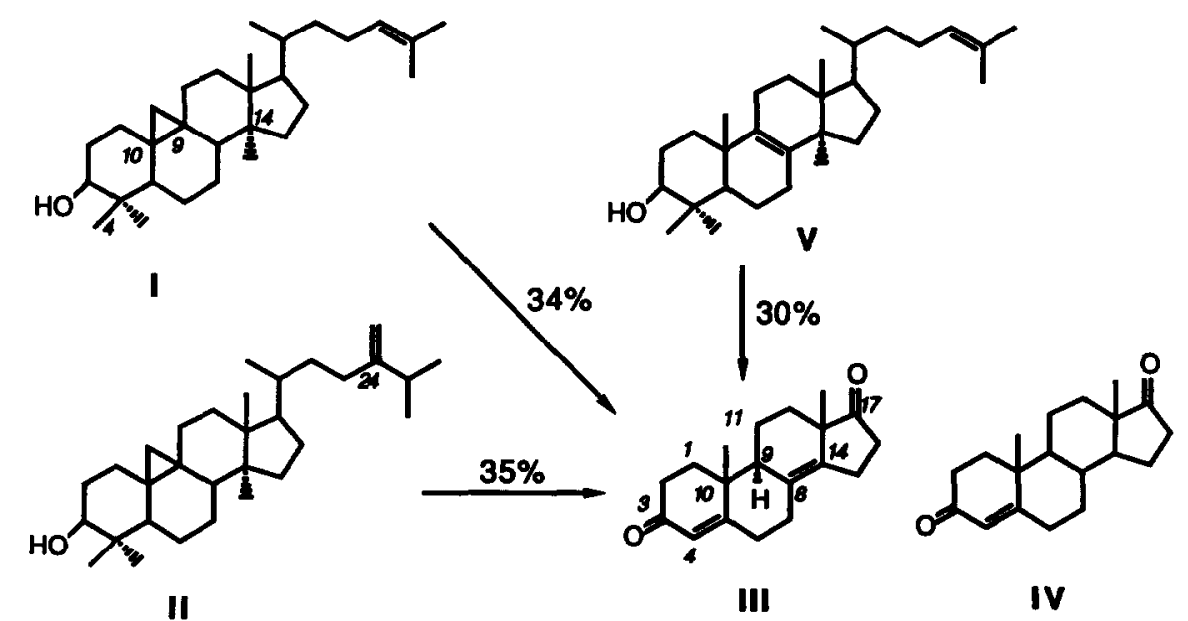


Compound III, $13 \mathrm{mp} .141-142^{\circ} \mathrm{C}$ from diethyl ether, shows in its high resolution mass spectrum a molecular ion at $\mathrm{m} / \mathrm{z} 284.2765$, corresponding to a formula of $\mathrm{C}_{19} \mathrm{H}_{24} \mathrm{O}_{2}$ (calcd. 284.1776). Its uv absorption maximum at $233.0 \mathrm{~nm}$ and ir absorption at $1669 \mathrm{~cm}^{-1}$ indicate an $\alpha, \beta$-unsaturated carbonyl chromophore. 14 Another ir absorption at $1742 \mathrm{~cm}^{-1}$ suggests the presence of a five-membered non-conjugated carbonyl. 14 These two structural moieties are supported by NMR spectra, of which the signal of an olefinic proton at $\delta 5.75$ (br. s, 4-H), and the carbon signals at $\delta 198.7$ (3-C), 169.6 (5-C) and 123.4 (4-C) constitute an $\alpha, \beta$-unsaturated carbonyl system, and the signal at $\delta 220.6$ represents the remaining non-conjugated carbonyl. In addition,

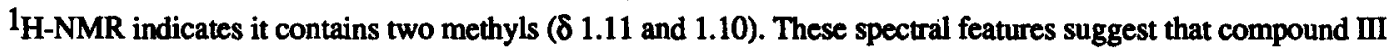
is an analogue of androst-4-ene-3,17-dione (IV) which was the major metabolite from the incubation of cholesterol with Mycobacterium sp (NRRL B-3805). 8 While comparing their formula, III contains two less hydrogens than IV, indicating that III has one more double bond equivalent . The 13C-NMR spectrum of III accounts for this difference by showing an extra fully substituted double bond ( $\delta 136.7$ and 127.8) and lacking two methines' signals in comparison with IV. From structural analysis, the fully substituted double bond in III can be only disposed between either C-8 and C-9 or between C-8 and C-14.

Several structures are possible to fit the above data. A hetero-long range COSY spectrum optimized for $J_{\mathrm{CH}}=10 \mathrm{~Hz}$ indicates that the methyl protons at $\delta 1.11$ is coupled to $17-\mathrm{C}(\delta 220.6), 14 \mathrm{C}$ (an olefinic carbon, $\delta 136.7$ ), 13-C (a quaternary carbon, $\delta 47.0$ ), and $12-\mathrm{C}$ (a methylene carbon, $\delta 28.7$ ), whereas the other methyl protons at $\delta 1.10$ is coupled to 5-C ( $\delta 169.6), 1-\mathrm{C}$ (a methylene carbon, $\delta 34.2)$ and 10-C (a quaternary carbon, $\delta 39.9)$. These data suggest that the fully substituted double bond is located between $C-8$ and C-14, and the two methyls ( $\delta 1.10$ and 1.11) are linked to C-10 and C-13, respectively. Thus, except the stereochemistry of C-9 remaining to be clarified, the skeleton of III is determined.

Chemical models indicate that if $9-\mathrm{H}(\delta 2.09$, br. t, $J=8.4 \mathrm{~Hz}$, designated from homo-COSY $)$ is $\alpha$-oriented, $11 \beta-\mathrm{H}(\delta 1.57, \mathrm{ddt}, J=14.0,14.0,10.3,3.6 \mathrm{~Hz}$, assigned from nOe studies and homo-COSY), a pseudoaxial proton, will be spatially close to $18-\mathrm{H}$ 's and 19-H's. Thus, nOe experiments upon irradiation either at $18-\mathrm{H}$ 's or 19-H's would enhance the intensity of $11 \beta-H$. On the other hand if $9-\mathrm{H}$ is $\beta$-oriented, the above nOe effects will not be observed. The actual nOe experiments show positive enhancements of $11 \beta-\mathrm{H}$ upon irradiation at 18-H's $(\delta 1.11)$ or 19-H's $(\delta 1.10)$. Consequently, 9-H is $\alpha$-oriented and compound III is androsta-4,8(14)diene-3,17-dione.

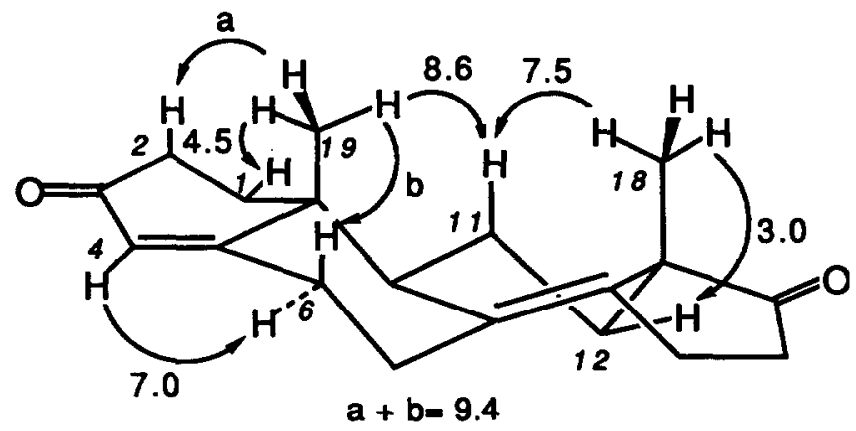

NOE's (\%) of compound III (CDCl 3 , Bruker $300 \mathrm{AM})$ 
This structure assignment is confirmed by single crystal X-ray crystallography. 15

Isolation of III from the incubation mixtures showed that the following changes had taken place: (i) the cyclopropyl ring was opened to form the C-19 methyl; (ii) three methyl groups located on the steroid nucleus were demethylated; (iii) two double bonds, $\Delta^{4}$ and $\Delta^{8(14)}$, were introduced, and (iv) the side chains were cleaved to give 17-ketone. These transformations except (iv) are first observed with Mycobacterium sp. (NRRL B-3805).

Lanosternl ( $\mathrm{V}$ ), an intermediate in the cholesterol biosynthesis with three methyl groups identical to those of I and II on the nucleus and has a $\Delta^{8(9)}$ double bond but without the cyclopropyl ring, appears to be an interesting substrate for transformation by this microorganism. Incubation of $\mathrm{V}$ with Mycobacterium sp. (NRRL B-3805) also gave compound III as the major metabolite isolable of $30 \%$ yield.

Currently, we are in the process of isolation and characterization of minor metabolites from the incubation mixtures for further information concerning this transformation.

Acknowledgment. We would like to thank Dr. Hsien-Jer Chen, Food Industry Research and Development Institute, Hsinchu, Taiwan, for the generous gift of crude oryzanol, Ms. Siew-Leng Ng of NSC northern NMR Instrumental Center for the measurement of 2D NMR and Mr. Gene-Hsiang Lee of NSC X-ray Center for the manipulation of X-ray data. We are grateful to NSC, R. O. C., for support of this research under Grant NSC 740606-B002-87 and NSC 78-0412-B002-14.

\section{References}

1. L. L. Smith in Biotechnology Vol. 6a. Biotransformation, 1984, p.31, edited by H. J. Rehm and G. Reed, Verlag Chemie.

2. K. Kieslich, J. Basic Microbiol., 1985, 25, 461.

3. C. J. Sih and H. W. Whitlock, Ann. Rev. Biochem. 1968, 37, 661.

4. (a) C. J. Sih and K. C. Wang, J. Amer. Chem. Soc., 1965, 87, 1387; (b) C. J. Sih, S. S. Lee, Y. Y. Tsong, K. C. Wang and F. N. Chang, ibid, 1965, 87, 2765.

5. G. Wix, K. G. Buki, E. Tomorkeny and G. Ambrus, Steroids, 1968, 11, 401.

6. (a) K. Arima, M. Nagasawa, N. Bae and G. Tamura, Agr. Biol. Chem., 1969, 33, 1636; (b) M. Nagasawa, M. Bae, G. Tamura and K. Arima, ibid, 1969, 33, 1644.

7. Van der W. F. Waard, J. Doodewarrd, de J. Flines and van der S. Weele, Abh. Deut. Akad. Wis. Berlin Kl. Med.,1969, 2, 101.

8. (a) W. J. Marsheck, S. Krachy and R. D. Muir, Appl. Microbiol, 1972, 23, 72; also (b) US Patent 1972, $3,684,657$ and $1973,3,759,791$.

9. M.G. Wovcha, F. J. Antosz, J. C. Knight, L.A. Komined and T. R. Pyke, Biochem. Biophys. Acta, 1978, $531,308$.

10. (a) C. J. Sih, K. C. Wang and H.H. Tai, J. Amer. Chem. Soc., 1967, 89, 1956 ; (b) C. J. Sih, H. H. Tai and Y. Y. Tsong, ibid., 1967, 89, 1957; (c) Y. Fujimoto, C. S. Chen, A. G. Gopalan and C. J. Sih, ibid, $1982,104,4718$; (d) Y. Fujimoto, C. S. Chen, A. G. Gopakan and C. J. Sih, ibid, 1982, 104, 4720.

11. T. Itoh, T. Tamura and T. Matsumoto, J. Amer. Oil. Chem. Soc., 1973, 50, 300.

12. The microorganism was maintained on a Bacteria Agar (Dextrose $1 \%$, yeast extract $1 \%$, Nutrient Broth $0.8 \%$ and Agar 3.5\%) at $260^{\circ} \mathrm{C}$ for 11 days then transfered and grown in Nutrient broth- Dextrose medium (Nutrient broth $1.6 \%$ and Dextrose $4 \%$ ) at $26-28^{\circ} \mathrm{C}$ on a rotary shaker (250 rpm, 1 -in stroke). Transformation was carried out in 2-L Erlenmyer flasks containing $400 \mathrm{ml}$ of medium. The substrate dissolved in DMF was added to the growing microorganism and the incubation was continued for 5 days.

13. The physical data of III were obtained from the following instruments: [ $\alpha$ ]D: JASCO DIP-181 Digital Polarimeter, m.p.: Buchi 510 Melting Apparatus; ir: Jasco A-100 Infrared Spectrophotometer; uv: Hitachi 15020 Double Beam Spectrophotometer, mass: Finnigan Mat 4500 series GCMS and Jeol JMS-HX110 Mass Spectrometer, NMR: Bruker AC-80 and AM-300. Some physical data of III: $[\alpha]_{\mathrm{D}}^{20}+316^{\circ}$ (c 0.25, EtOH); 
ir: $\lambda_{\max }\left(\mathrm{KBr}, \mathrm{cm}^{-1)} 2961,1742,1669,1610,1447,1370\right.$; uv : $\lambda_{\max }(\mathrm{MeOH}, \log \varepsilon) 233.0 \mathrm{~nm}(4.07)$; EIMS: $\mathrm{m} / \mathrm{z}$ (\%) $285\left([\mathrm{M}+1]^{+}\right.$, , 68.2), $284\left(\mathrm{M}^{+}, \mathrm{100.0}\right), 227(24.3), 212$ (81.0), 209 (61.0), 199 (22.1); 1 H-NMR assignment: $1.80(1 \alpha), 1.95(1 \beta), 2.35(2 \alpha$ and $2 \beta), 5.75(4), 2.39(6 \alpha$ and $6 \beta), 1.91(7 \alpha), 2.71$ (7ß), $2.09(9), 1.73(11 \alpha), 1.57(11 \beta), 1.21(12 \alpha), 1.78(12 \beta), 2.18(15 \alpha), 2.58(15 \beta), 2.78(16 \alpha), 2.55$ (16B), 1.11 (18), 1.10 (19). 13C-NMR assignment: 34.2 (C-1), 33.8 (C-2), 198.7 (C-3), 123.4 (C-4), 169.6 (C-5), 32.3 (C-6), 28.8 (C-7), 127.8 (C-8), 47.6 (C-9), 39.9 (C-10), 18.4 (C-11), 28.7 (C-12), 47.0 (C-13), 136.7 (C-14), 35.8 (C-15), 22.9 (C-16), 220.6 (C-17), 21.8 (C-18) and 18.2 (C-19). These assignments were made by nOe and 2D NMR techniques and will be described somewhere else in more details.

14. K. Nakanishi, Infrared Absorption Spectroscopy, 1976, p.42, 1st ed, Holden-Day, San Francisco.

15. Crystal data of compound III: orthorhombic P 2,2,2; $a=8.994$ (3), $b=10.483$ (2), $c=16.680$ (4) A, $Z=4$, Intensity data were collected on a CAD-4 diffractometer with $\theta / 2 \theta$ scan mode, using monochromated MoK $\alpha$ radiation. Data were measured up to $2 \theta$ of $50^{\circ}$. A total of 1607 reflections were collected. Among them, 637 were considered to be observed $(>1.5 \sigma(I)$ ). Final agreement indices are $R(F)=0.064, W R(F)=0.056, S=$ 2.7, based on anisotropic refinement of all non-hydrogen atoms. The list of atomic co-ordinates and molecular dimensions have been deposited with Cambridge Crystallographic Data Center, University Chemical Laboratory, Lensfield Road, Cambridge CB2 IEW.

(Received in Japan 25 December 1989) 